\title{
37. The Early Evolution of the Solar System
}

\author{
A. G. W. Cameron \\ Yeshiva University and \\ Goddard Institute for Space Studies, NASA \\ New York, New York
}

\begin{abstract}
The problems of relating collapse conditions in an interstellar cloud to a model of the primitive solar nebula are discussed. In such a nebula there is a radial force balance between gravity, the pressure gradient, and centrifugal forces due to the rotation. Approximate values are given for the combinations of temperature and density throughout the nebula, from a maximum of about $2000^{\circ} \mathrm{K}$ near the center to less than $200^{\circ} \mathrm{K}$ in the outer portion. These conditions are based upon the compression adiabats in the terminal stages of the collapse of an interstellar cloud. One general conclusion, of great importance for accumulation of bodies within the solar system, is that interstellar grains should not be completely evaporated at distances in the nebula beyond about one or two astronomical units.
\end{abstract}

$\mathrm{D}$ URING THE LAST FEW YEARS, I have been attempting to construct quantitative numerical models of the early solar nebula, which take into account some of the physics of star formation (for refs. see Cameron, 1970). It appears that star formation is initiated when a typical interstellar cloud undergoes collapse as a result of a prior compression to a higher-thannormal density in the interstellar medium. This compression can result in part from the shock transition undergone by the gas when it flows into the spiral arm of the galaxy, and it can also occur when hot stars, newly formed in the vicinity, reach the main sequence and ionize the surface layers of the interstellar cloud. Under these circumstances, the local pressure in the vicinity of the cloud becomes considerably increased, so that the interior of the cloud can become compressed and a dynamical collapse under the force of its own gravity can take place.

During the collapse of the cloud the interior becomes exceedingly cold. This results in part from a diminishing of heating by starlight and possibly by interstellar cosmic rays, and in part from the increased cooling efficiency of the gas. Temperatures of the order of 5 or $10^{\circ} \mathrm{K}$ may be reached. At these temperatures the speed of sound in the cloud is very small, so that pressure waves are unable to transmit from one part of the cloud to a more distant part any information about the current state of collapse of the first part of the cloud. This promotes the fragmentation of the cloud into smaller pieces, and progressive fragmentation should take place as the collapse proceeds.

The question of the angular momentum to be associated with the cloud fragments is a complicated problem. Since the collapse of the cloud should be initiated by compressive processes, then a great deal of turbulence should be present in it from the beginning of the collapse. The kinetic energy of the turbulence acts like a gas which compresses with a ratio of specific heats of 5,3 , while at the same time the temperature in the interior is decreasing as a result of the enhanced cooling efficiency. Hence, the relative energy 
content in the turbulence will progressively increase until typical turbulent velocities become sonic. If these velocities were to become supersonic, they would be rapidly dissipated by shock phenomena. Hence the total internal energy in the form of turbulence will be comparable to the thermal energy of the cloud during the collapse phase. There will be a random turbulent component of the angular momentum in any given fragment of the cloud, which is likely to exceed the angular momentum that the fragment would have as a result of the initial rotation of the cloud itself. The turbulent component of the angular momentum will undergo a wide range of statistical variation.

Another complicating feature will be some redistribution of the angular momentum within a cloud fragment. A fragment presumably is formed as a result of a density fluctuation in the cloud, and hence it forms about some spot in the cloud where the density reaches a local maximum. As the fragment continues to collapse, the center will collapse more rapidly than the periphery of the fragment, so that the center will achieve a higher angular velocity than the periphery, and hence there can be a turbulent transfer of angular momentum from the central region to the exterior as the fragment collapse proceeds.

These general considerations lead me to believe that the fragments of the gas cloud will have both a wide variation in their total angular momentum, and also a considerable variation in the internal distribution of their angular momentum. Hence we have no good a priori basis for choosing a particular distribution of angular momentum within such a fragment, nor a total angular momentum for a fragment, in constructing any theory of the formation of the solar system. One should construct a theory which contains the angular momentum distribution as a parameter, or set of parameters, and then see whether the solar system corresponds to a particular set of such parameters. I believe that the model discussed below contains a reasonable angular momentum content to correspond to solar system conditions, but the theory described is incomplete, since at the present time the model is being used as a vehicle for the development of the necessary physical input toward further calculations.

The models which have been calculated have been based upon a spherical distribution of matter representing a fragment of an interstellar cloud. This spherical distribution of matter has been taken to be uniformly rotating, but it need not have a uniform density distribution in the interior. In fact, two cases have been taken: a uniform internal density distribution, and a density distribution which varies linearly from a central value to zero at the surface of the sphere. The model shown in figure 1 is based upon the latter assumption, but there is not a great deal of difference between the models which result from these assumptions. The total mass in the initial sphere, which is taken to collapse down to a thin disk, is two solar masses. This allows for the loss of a considerable amount of mass from the Sun in the T-Tauri phase solar wind, and also for the presence of mass in the nebula which will not be incorporated in the Sun, but which will be carried away by the T-Tauri solar wind.

When this program was started about three or four years ago, my first approach was to assume that the nebula would be very thin, so that the radial properties of the solar nebula could be considered as disconnected from the vertical properties. The radial properties were taken to be given

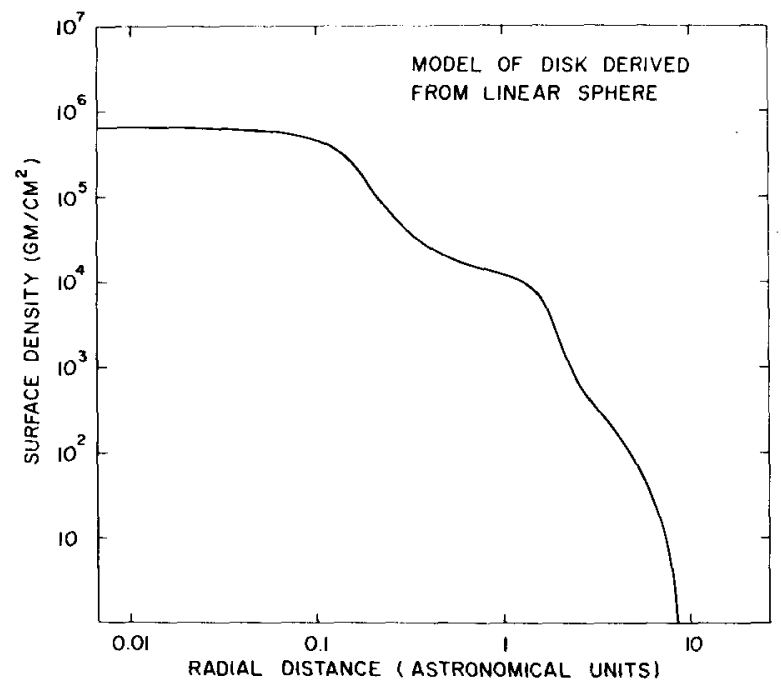

Figure 1.-Surface density of a two solar mass model of the primitive solar nebula, derived from a uniformly rotating sphere with a density varying linearly from a central value to zero at the surface. The radial pressure gradient has not been included in the force balance. 
by a balance between gravity and the centrifugal force due to rotation, and the vertical properties were taken to be hydrostatic equilibrium, with a balance between gravity and the pressure gradient. In the latter case, any point in the nebula was considered to be part of an infinite plane having the same properties everywhere. While this proved to be a useful first orientation to the problem, both of the assumptions have proven to be inadequate, and at the present time the problem is being carried out with a partial recoupling of the vertical and radial properties in the disk.

The radial force balance in the disk is given by the following expression:

$$
-\left\langle\frac{1}{\rho} \frac{d P}{d r}\right\rangle+r \Omega^{2}-\frac{d \varphi}{d r}=0
$$

in which the terms represent, respectively, the average pressure gradient in the radial direction, the centrifugal force per unit mass, and the gravitational force per unit mass; $\rho$ is the density, $P$ the pressure, $r$ the radial distance, $\Omega$ the angular velocity, and $\varphi$ the gravitational potential.

For the vertical structure, if there were an infinite plane with the same properties everywhere, then the gravitational potential would be given by the following equation:

$$
\frac{d^{2} \varphi}{d z^{2}}=-4 \pi G \rho
$$

The hydrostatic equation, utilizing the perfect gas law, may be written in the following form:

$$
-\frac{1}{\rho} \frac{d P}{d z}=\frac{N_{0} k T}{\mu \rho} \frac{d \rho}{d z} \approx \frac{d \varphi}{d z}+\frac{z}{r} \frac{d \varphi}{d r}
$$

In the right hand side of this equation, we take the first term to be given by the solution of equation (2) for an infinite plane, and the second term is intended to represent the component of force due to a central gas concentration in the nebula which would be obtained from the equilibrium in the radial direction. The temperature gradient in the vertical direction is given by the requirement that energy be transported from the interior of the nebula to the surface, where it can be radiated away at the effective surface temperature. Radiative transfer will suffice to do this if the resulting temperature gradient proves to be subadiabatic, in which case we have

$$
\frac{d T}{d z}=-\frac{3}{4 a c} \frac{\kappa \rho}{T^{3}} L
$$

On the other hand, if radiative transfer would require a superadiabatic gradient, then convection will set in to transport energy in the vertical direction, and we can then assume an adiabatic temperature gradient:

$$
\frac{d T}{d z}=\left(1-\frac{1}{\gamma}\right) \frac{T}{P} \frac{d P}{d z}
$$

In these equations $T$ is temperature, $a$ is the Stefan-Boltzmann constant, $c$ is the velocity of light, $\kappa$ is the radiative opacity, $L$ is the luminosity or rate of energy transfer, and $\gamma$ is the ratio of specific heats. The rate of change of luminosity is equal to the rate at which gravitational potential energy is locally released by the contraction of the disk in the vertical direction, and is given by

$$
\frac{d L}{d z}=-\rho\left(\frac{d \varphi}{d z}+\frac{z}{r} \frac{d \varphi}{d r}\right) \frac{d z}{d t}
$$

The solution of the vertical structure equations constitutes an eigenvalue problem, similar to that involved in the construction of a model of the stellar interior. Strictly speaking, one should follow the detailed evolution of every point in the nebula as one does for the evolution of a star, but this is far too complicated for the present approach. Instead, we assume that the relative height of the nebula at any point changes in a homologous fashion, as indicated by the following relation:

$$
\frac{1}{z} \frac{d z}{d t}=\text { const }
$$

This allows the eigenvalue problem for the vertical structure to be solved independently of the previous history of the material.

At present the numerical solutions for the radial and vertical structure in the model of the primitive solar nebula are being carried out by Mr. Milton Pine, a graduate student at Yeshiva University. We have not yet succeeded in obtaining a full solution to the problem of the initial structure of the nebula, but we are close to it, and some of the numbers that I shall report here can be taken as correct in order of magnitude for such a model.

Figure 1 shows the structure of the two solar 
mass disk in the absence of radial pressure gradients. This is the disk corresponding to the flattening of a uniformly rotating sphere in which the density varies linearly from the central value to zero at the surface. The angular momentum contained in the model happens to correspond to an assumption of corotation of the initial interstellar cloud, at the threshold of gravitational collapse, with the motion of the cloud about the center of the galaxy.

In this figure is plotted the surface density of the nebula in grams per square centimeter as a function of the radial distance in astronomical units. Notice that the dimension of the disk is comparable to the size of the solar system, so that this sort of model does not misrepresent the primitive solar nebula too badly. The surface density near the center of the disk is so small that there is no effective central body which can be called a star in the nebula. There is only a mild central density concentration in the disk.

Throughout the disk, except in the very central region, there is a large amount of shear. In this very wide range of radial distance the tangential velocity in the gas is roughly the same, about $10 \mathrm{~km} / \mathrm{s}$, so that the corresponding angular velocity varies roughly inversely as the radial distance.

This disk model is entirely two-dimensional in character. It is well known that disks of zero thickness are highly unstable against both radial and nonradial perturbations, so that rings would form in such disks if they were given a chance to do so. The method of calculation used to construct the model shown in figure 1 tends to suppress local ring instabilities in the disk but the convergence to the solution shown in the figure is a very slow one even on a very fast computer. In our earliest work I found rings in the solution near the exterior portion of the disk. These rings turned out to be solutions to the difference equations which resulted from the finite zoning used to construct the model, but they were not actual solutions to the problem. When the size of the zones was decreased, the rings disappeared. If the thickness of the nebula is sufficiently great, then the radial and nonradial instabilities are expected to be suppressed, so that no rings would be present.

The next step, which we initiated recently, was to add the radial gas pressure gradient to the force balance in the radial direction. When that was done, we found that the mathematical technique used to produce the gravitational selfconsistent model in figure 1 no longer seemed to produce convergence at all. Thus in the last few months we have abandoned this method, and we have started to calculate the potential for a finite three-dimensional disk, taking into account the actual height of the disk at all points. The motivation in doing this has been to decrease the dependence of the potential calculation upon fluctuations in density on local distance scales, which tend to introduce instabilities into the problem. This potential calculation is considerably lengthier than it was in our two-dimensional approximation, and hence we have been seeking a method for solving the structure equations which does not involve an iteration procedure with matrix inversion at every step. We have not yet fully succeeded in accomplishing this, but we have produced models with approximate force balance, and these can be used to give some approximate ideas of the physical conditions to be expected in the initial solar nebula.

The introduction of the radial pressure gradient causes an expansion of the disk in the radial direction. This expansion is greater near the center of the disk than it is near the outside, and the central density is lowered to something in the vicinity of $10^{5}$ grams per square centimeter column.

In order to determine the temperature gradient in the vertical direction, we need values for the radiative opacity. This opacity is a function of temperature and density. At the highest temperatures of interest in the nebula, opacities calculated for stellar interiors are available. At lower temperatures, the opacity is mainly due to small iron grains. The electrical conductivity of metallic iron allows the absorption of electromagnetic radiation of all wavelengths. Fortunately, the size of the grains is not important as long as the grains are small compared to the interesting wavelengths, since the opacity depends upon the total volume of the grains and not upon their total surface area. At higher pressures such triatomic molecules as water make an important contribution to the opacity, but these pressures are not encountered significantly in the model of the 
initial solar nebula which we have constructed. The opacity which we have computed for this problem is shown in figure 2. The rapid changes in opacity occur at temperature and density combination where the iron grains condense out of the vapor phase. We have not taken into account the reduction in opacity that would occur if the iron should be transformed into magnetite at a temperature in the vicinity of $500^{\circ} \mathrm{K}$.

One point of considerable interest is the minimum surface density required for convection to exist in the disk. We have calculated this as a function of the central temperature for the assumption that the vertical structure corresponds to a local portion of an infinite plane. This critical minimum surface density is shown in figure 3 . The opacity of the iron grains dominates the determination of this critical density up to a temperature of about $1500^{\circ} \mathrm{K}$. Above this, the iron disappears, and the opacity is mainly due to the $\mathrm{H}^{-}$ion. Above $2000^{\circ} \mathrm{K}$, the dissociation of hydrogen molecules sets in, which lowers the effective ratio of specific heats, so that the critical surface density is very considerably lowered once again, and it only recovers very slowly toward higher temperatures as shown in figure 3. It may be seen that in the lower temperature region, the critical surface density needed for convection is comparable to the surface densities throughout the inner regions of the disk.

There are a number of powerful dissipation mechanisms which will operate in the primordial solar nebula. In a convecting region, the very large turbulent viscosity due to the convection will cause a fairly rapid outward transport of angular momentum, which must be accompanied by an inward transport of matter and a fairly significant additional release of gravitational potential energy. However, the pressure in the middle of the plane is much greater than that in the upper vertical layers of the disk, and hence the pressure gradient near the middle is also greater than the pressure gradient near the surface. Consequently, a total force balance requires that the upper levels of the disk be rotating more rapidly than the central layers. This refers to any particular radial distance in the disk. However, in a convecting region it is required that the gas should approach a condition where the angular velocity is constant on a cylindrical surface

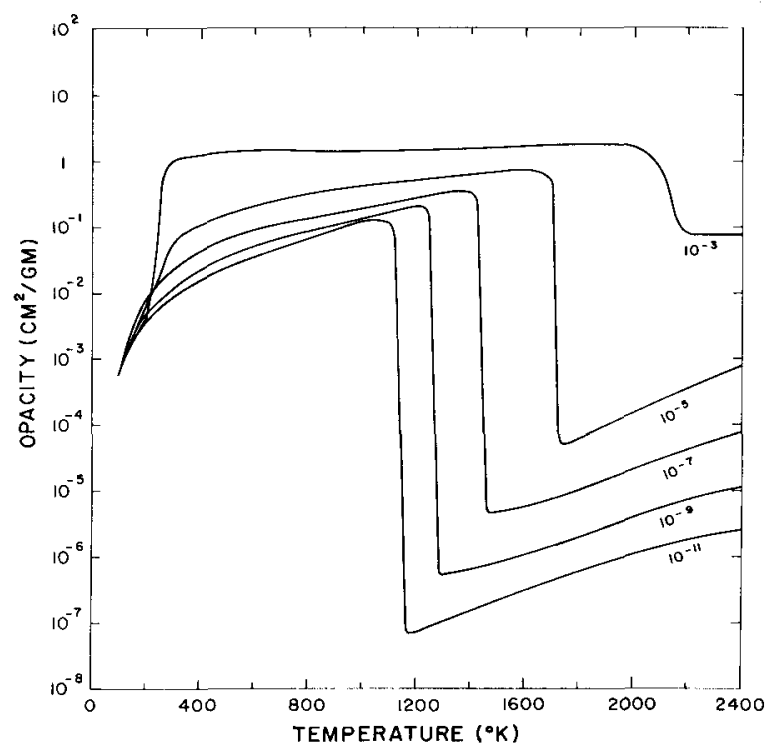

Figure 2.-Opacity of material of solar composition as a function of temperature for various pressures, assuming that the condensed iron grains are small compared to the important thermal wavelengths of light and that no transformation to magnetite occurs at low temperatures.

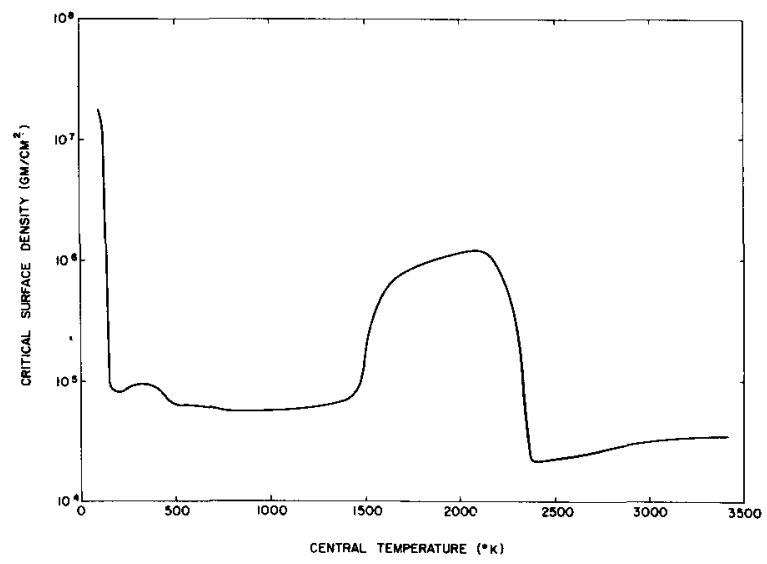

Figure 3.-Minimum surface densities required for convection, as a function of central temperature, for a part of an infinite plane distribution of material.

centered on the axis of rotation of the disk. This will give rise to large-scale currents in a convection zone which will rise and flow away from the center at high levels and will fall and flow inward near the center of the disk. These currents will also contribute to the rapid outward transport of angular momentum in the disk. 
In a radiative region other effects take place. Here there is a force balance involving gas pressure gradients, centrifugal forces, and gravitational potential gradients. The "level surfaces" are not perpendicular to the gravitational potential gradients. Nevertheless, for a completely static situation we would require that the pressure, density, and temperature should all be constant on level surfaces. The divergence of the radiative energy flux should also be zero everywhere on a level surface, in the absence of internal energy sources. However, it is not possible to satisfy all of these conditions, and hence we cannot have a fully static solution. The divergence of the radiative energy flux can only be constant on the average over a level surface. Nearer the axis of rotation, where the temperature is higher, the excess temperature gradient will give rise to an outward flowing motion of gas, in which the divergence of the energy flux is balanced by the adiabatic expansion of the gas. Near the mid-plane of the disk on the same level surface, the negative value of the divergence of the energy flux is balanced by the adiabatic compression of the gas. This gives rise to Eddington-Sweet circulation currents. The theory of such currents has been discussed only in the limit where the centrifugal force perturbations are small compared to gravity, not comparable as in the present case. A proper theory for the present case would require full development of higher order terms, and this has not been carried out. However, I estimate that the Eddington-Sweet circulation currents in the radiative part of the solar nebula probably have velocities which are a substantial fraction of the velocity of sound.
All of these dissipation mechanisms lead to a rapid outward transport of angular momentum, and a very short dissipation time for the gas in the disk. Hence we must expect that whatever accumulation of large bodies is to take place with the assistance of the gas in the disk, must take place quickly before the Sun is formed by the dissipation of the disk, and the T-Tauri phase solar wind then sweeps away the remaining gas.

Table 1 shows some rough estimates for the physical conditions in the initial solar nebula. The combinations of temperature and density shown in the table are intended to represent values at the center of the disk. These combinations of temperature and density correspond to the final compressive adiabat to be expected in the interior of a fragment of an interstellar cloud as estimated by Larson (1969). In using this adiabat, I have ignored the alterations in it that might be produced by the shock which decelerates the material as it falls inward to form the disk through collapse of the interstellar gas fragment.

As shown in table 1, the temperature will have a maximum value in the vicinity of $2000^{\circ} \mathrm{K}$ near the center of the nebula, and will progressively decrease until a value of less than $200^{\circ} \mathrm{K}$ is reached near the outer part of the disk. The central pressure is about one millibar, and decreases to about a microbar in the outer parts of the disk. The thickness of the disk near the center is about half an $\mathrm{AU}$, and this increases to become slightly more than an astronomical unit in the outer portion. Perhaps of greatest interest in terms of the accumulation of bodies in the disk are the ratios of the radial pressure gradient to the centrifugal forces at the mid-point in the plane of the disk.

TaBle 1.-Very Approximate Initial Conditions in the Solar Nebula

\begin{tabular}{|c|c|c|c|c|c|}
\hline Radial distance & Surface density & Temperature & Pressure & Thickness & $\left(1 \frac{d P_{c}}{}\right)$ \\
\hline$(\mathrm{AU})$ & $\left(\mathrm{g} / \mathrm{cm}^{2}\right)$ & $\left({ }^{\circ} \mathbf{K}\right)$ & (atm) & $(\mathrm{AU})$ & $r \Omega^{2}$ \\
\hline 0 & $1 \times 10^{5}$ & 2000 & $1 \times 10^{-3}$ & 0.5 & 1 \\
\hline 1 & $5 \times 10^{4}$ & 1500 & $3 \times 10^{-4}$ & 0.6 & 0.5 \\
\hline 5 & $1.8 \times 10^{4}$ & 640 & $3 \times 10^{-5}$ & 0.8 & 0.1 \\
\hline 20 & $4 \times 10^{3}$ & 170 & $1.5 \times 10^{-6}$ & 1.1 & 0.03 \\
\hline
\end{tabular}


This ratio is near unity near the center of the disk, and is still several percent in the outer portions. The significance in this ratio lies in the fact that the gas in the disk, which is partially supported by a pressure gradient, will rotate at less than Keplerian orbital velocity, whereas solid particles of significant size in the disk will not be supported by the gas radial pressure gradient, and hence will suffer a continuing gas drag, and will gradually spiral in toward the center of the nebula. This would be a general tendency which would be superimposed upon the migrations of the particles produced by the fairly strong currents which would exist in different parts of the disk.

The disk temperatures shown are probably comparable to the highest temperatures to which particles will be subjected in various parts of the disk. The disk will cool as a function of time, thus lowering the temperatures over the initial values shown in table 1. At the center of the disk, the temperature is high enough to evaporate iron and silicate materials, and hence we would expect that the interstellar grains which accompany the gas in its collapse would be destroyed inside a radial distance of 1 or $2 \mathrm{AU}$. However, the grains will not be completely evaporated at larger radial distances in the disk. The more volatile substances will be evaporated from them, but they will remain as centers of condensation for these volatiles when the temperature falls once again.
Sulfur should remain in the form of troilite at distances of about $5 \mathrm{AU}$ and greater. The common ices should remain in the grains at distances of the order of $20 \mathrm{AU}$ and greater. This is where we should expect cometary accumulation to occur, and it is likely that cometary accumulation occurring at $20 \mathrm{AU}$ and beyond has been responsible for providing the basic material which led to the accumulation of the planets Uranus and Neptune. I would not expect the ices to be present in the particles which would initially accumulate at the distance of the asteroids, Jupiter, and Saturn. I believe it more likely that rocky bodies should form at these distances, and grow in size until they are able to capture appreciable amounts of gas from the solar nebula, thus leading eventually to bodies which largely resemble the Sun in composition. In the inner solar system the large turbulent and circulatory motions of the gas are probably sufficient to limit greatly the amount of gas accumulation that can take place on forming bodies, and the subsequent T-Tauri phase solar wind should then be able to remove not-toomassive primitive atmospheres on the inner terrestrial planets.

\section{ACKNOWLEDGMENT}

This work has been supported in part by grants from the National Science Foundation and the National Aeronautics and Space Administration.

\section{REFERENCES}

Cameron, A. G. W., 1970. Formation of the Earth-Moon system, EOS, Trans. Am. Geophys. Union, 51, 628-633.

LARson, R. B., 1969. Numerical calculations of the dynamics of a collapsing proto-star, Monthly Notices Roy. Astron. Soc., 145, 271-295. 